\title{
El tamaño del cladodio y los niveles de auxina influyen en la propagación asexual de pitaya (Selenicereus megalanthus Haw.)
}

\author{
Effect of different cladode size and auxin levels in asexual \\ propagation of pitaya (Selenicereus megalanthus Haw.)
}

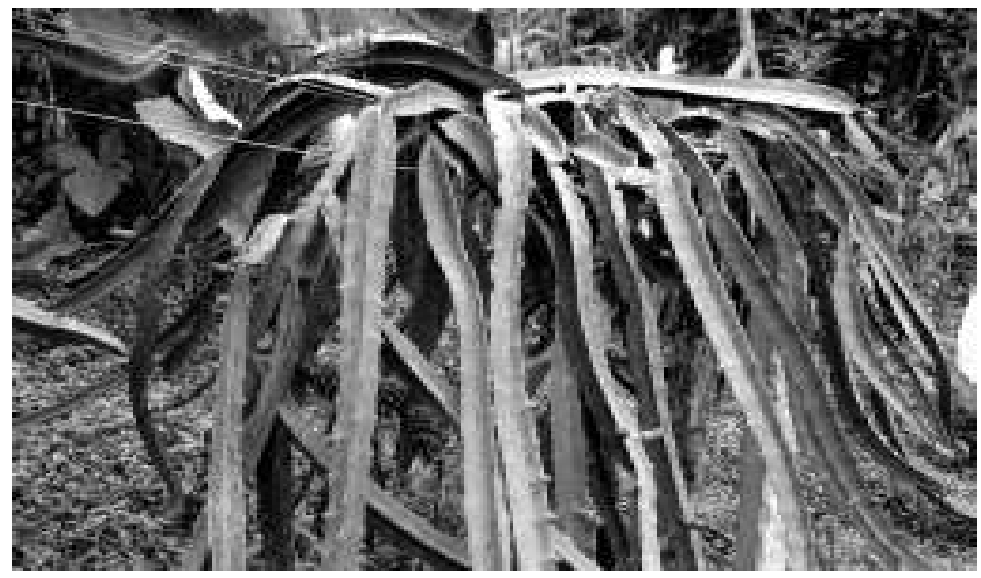

HELBER ENRIQUE BALAGUERA-LÓPEZ ${ }^{1,3}$

EMERSON IVÁN MORALES ${ }^{1}$

PEDRO JOSÉ ALMANZA-MERCHÁN²

WILLIAM ALBERTO BALAGUERA L.'

Planta adulta de pitaya.

Foto: H.E. Balaguera-López

\section{RESUMEN}

La pitaya es una fruta de gran aceptación en los mercados nacionales e internacionales, debido a su exótico sabor dulce y refrescante y a sus propiedades medicinales. Comercialmente, la pitaya es propagada asexualmente mediante estacas o cladodios, sin embargo, los estudios y las técnicas al respecto, que garanticen la obtención de plantas con un sistema radical abundante, uniforme y de buena calidad, son muy pocos, por lo cual se presentan problemas como bajo prendimiento en campo, retardo en la producción, baja producción y corta vida útil de las plantas. En el presente estudio se evaluó el efecto de cuatro concentraciones de ácido indolbutírico (AIB) $\left(0,1.500,3.000\right.$ ó $\left.4.500 \mathrm{mg} \mathrm{L}^{-1}\right)$ y de dos tamaños de cladodio (40 ó $60 \mathrm{~cm}$ de altura) en la propagación asexual de pitaya durante 90 días; se utilizó un diseño completamente al azar. Se encontró que la aplicación de $4.500 \mathrm{mg} \mathrm{L}^{-1}$ de AIB a las estacas de $60 \mathrm{~cm}$, como tratamiento o como factores independientes, garantizaron los mejores resultados al generar mayor enraizamiento, número de raíces y brotes, longitud de raíces y brotes, masa fresca y seca de raíz y brotes; por lo tanto, este tratamiento es recomendable para la propagación asexual de pitaya.

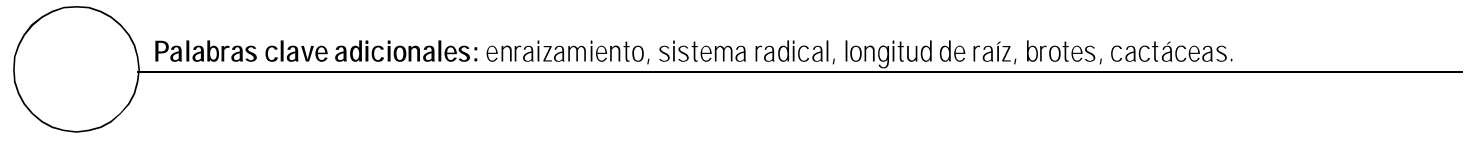

\footnotetext{
Facultad de Ciencias Agropecuarias, Grupo de Investigaciones Agrícolas, Universidad Pedagógica y Tecnológica de Colombia (UPTC), Tunja (Colombia).

2 Facultad de Ciencias Agropecuarias, Grupo Ecofisiología Vegetal, Universidad Pedagógica y Tecnológica de Colombia (UPTC), Tunja (Colombia).

3 Autor para correspondencia.enrique_balaguera@yahoo.com
} 


\begin{abstract}
Pitaya is a highly appreciated fruit in the national and international markets due to its exotic, sweet and refreshing flavor and medicinal properties. In commercial production, pitaya is asexually propagated by cuttings or cladodes, but there are few studies or techniques related to propagation that improves the obtaining of plants with abundant uniform root systems and that are of high quality; therefore, there are problems, such as low rates of rooting, which retard yield and shorten the duration of the crop. In this study, the effect of four concentrations of indole butyric acid (IBA) $\left(0 ; 1,500 ; 3,000\right.$, and 4,500 $\left.\mathrm{mg} \mathrm{L}^{-1}\right)$ and two cladode sizes (40 and 60 $\mathrm{cm}$ in height) on asexual propagation of pitaya over the course of 90 days was evaluated; a completely randomized design was used. It was found that application of 4,500 mg L-1 IBA to the cladode of $60 \mathrm{~cm}$ in height as a treatment or as an independent factor ensured the best results by generating increased rooting, number of roots and shoots, length of roots and shoots, fresh and dry mass of roots and shoots. Therefore, this treatment is recommended for asexual propagation of pitaya.
\end{abstract}

Additional keywords: rooting, root system, root length, shoots, cacti.

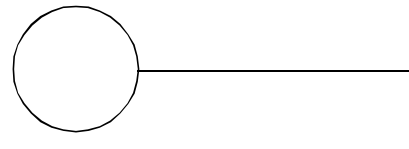

La pitaya amarilla (Selenicereus megalanthus Haw.) es una fruta tropical originaria de América, cuyos principales productores mundiales son Colombia y México; es una fruta exótica muy apetecida para su consumo en diversos países del mundo, gracias a su contenido nutricional, sabor y aroma, así como por sus propiedades biofuncionales y medicinales (Dueñas et al., 2008). En octubre de 2006, Colombia exportó 5,8 t de pitaya amarilla, y entre los meses de enero a octubre, un total de 117 t, con importantes volúmenes a la Unión Europea y a Estados Unidos, entre otros $(\mathrm{CCl}$, 2008). Para el 2008, Colombia tenía un área sembrada de 478 ha, con una producción de 3.989 t y un rendimiento de 8,3 tha $^{-1}$ (Agronet, 2010).

Comercialmente, la pitaya es propagada asexualmente mediante cladodios maduros 0 estacas, sin embargo, no se ha logrado obtener una técnica de propagación que permita la formación de un sistema radical uniforme, abundante, vigoroso y en poco tiempo, lo cual ha generado que las plantas tarden más tiempo en adaptarse a las condiciones de campo y sean menos competitivas en condiciones adversas, lo que genera bajo prendimiento, plantas que tardan bastante en llegar a producción, cosechas irregulares y menor vida útil de la planta.

Con la utilización de promotores del crecimiento vegetal, como el ácido indolbutírico (AIB), es posible aumentar y acelerar la formación de raíces adventicias en la propagación asexual de plantas (Moreno et al., 2009; Li et al., 2009). Para el enraizamiento es necesario un balance hormonal entre promotores e inhibidores de iniciación radicular (Pasqual et al., 2001), lo cual se puede lograr con la aplicación exógena de AIB, ya que elevan la concentración de auxinas en el tejido tratado (Hinojosa, 2000).

En horticultura, el efecto estimulador de las auxinas en la formación de raíces adventicias ha sido utilizado con éxito para la propagación asexual (Taiz y Zeiger, 2006). La formación de raíces adventicias sigue fases muy similares y comparte mecanismos comunes con la formación de raíces laterales (Ermel et al., 2000); este proceso consta de tres fases: inducción, iniciación y expresión. La inducción comprende eventos moleculares y bioquímicos sin cambios visibles; la iniciación se caracteriza por la división celular y organización del primordio radical, y la expresión consiste en el crecimiento del primordio radical dentro del tal lo y la emergencia de las raíces (Li et al., 2009; Ermel et al., 2000). Asimismo, se propuso un modelo en el que se necesita el ácido indolacético (AIA) al menos en dos etapas de la formación de las raíces laterales; en la primera, el AIA es transportado acropétalamente a la estela donde es requerido 
para iniciar la división celular en el periciclo, y en la segunda, se necesita el AIA para promover la división celular y mantener la viabilidad de las células durante el desarrollo de las raíces laterales (Celenza et al. 1995).

Bastos et al. (2006) encontraron en pitaya roja (Hylocereus undatus) mayor porcentaje de estacas enraizadas y de raíces emitidas por estaca con la utilización de $3.000 \mathrm{mg} \cdot \mathrm{L}^{-1}$, comparado con un testigo sin aplicación. Resultados similares, con la aplicación de AIB, fueron observados por Castrillón et al. (2008), en Vaccinium meridionale Swartz, y Santelices (2007), en Nothofagus glauca (Phil.).

La propagación por estacas es un método asexual cuya viabilidad depende de la capacidad de formación de raíces, de la calidad del sistema radical formado y del posterior desarrollo en campo de la planta propagada (Fachinello et al., 1995). Según López-Gómez et al. (2000), el tamaño del cladodio, entre otros factores, también tiene influencia sobre el poder de enraizamiento en cactáceas, principalmente pitaya, mediante la producción y translocación de hormonas y carbohidratos necesarios para la formación de raíces.

Mizrahi et al. (2002) afirman que la pitaya se debe propagar con estacas entre 25 y $40 \mathrm{~cm}$ de longitud, mientras que Bastos et al. (2006) encontraron una respuesta más favorable con estacas de $25 \mathrm{~cm}$ respecto a estacas de $15 \mathrm{~cm}$. No obstante, LópezGómez et al. (2000) recomiendan propagar pitaya roja de la especie Stenocereus griseus, cultivada en México, utilizando tallos o estacas de $0,5 \mathrm{~m}$ de longitud con mejores resultados y ahorro de material respecto a tallos de $1 \mathrm{~m}$; este material va con la parte apical cortada y plantados en posición vertical.

El enraizamiento también depende de las sustancias de reserva y la concentración hormonal que presente el material de propagación; la presencia de hojas tiene una fuerte influencia en el proceso de rizogénesis (Hartmann et al., 2002), ya que son tejidos ricos en auxinas, por lo que, probablemente, el éxito del enraizamiento depende en gran medida de la presencia de este órgano (Santelices, 2007). Por otra parte, de acuerdo con lo informado por Costa y Challa (2002), la superficie foliar en las estacas puede llegar a ser un indicador de la cantidad de raíces producidas. No obstante, en pitaya la función de las hojas (fotosíntesis, producción de ciertas hormonas, entre otras.) es relegada a los tallos o cladodios, por lo cual, los efectos anteriormente mencionados pueden estar relacionados con el tamaño del cladodio.

El objetivo de este estudio fue evaluar el efecto de diferentes concentraciones de AIB y de dos tamaños de cladodio en el enraizamiento de estacas de pitaya, con el fin de obtener plantas listas para llevar a campo con un sistema radicular de calidad que garantice altas producciones en el cultivo.

\section{MATERIALES Y MÉTODOS}

El experimento se realizó en el invernadero de vidrio de la Facultad de Ciencias Agropecuarias de la Universidad Pedagógica y Tecnológica de Colombia, en la ciudad de Tunja (Boyacá), la cual está localizada a $5^{\circ} 33^{\prime} \mathrm{N}$ y $73^{\circ} 24^{\prime} \mathrm{W}$ y a una altitud de $2.690 \mathrm{msnm}$; la temperatura promedio interna fue de aproximadamente $20^{\circ} \mathrm{C}$, y la humedad relativa, del $80 \%$. Se utilizó un diseño completamente al azar bifactorial de $4 \times 2$, donde el primer factor correspondió a la aplicación de diferentes concentraciones de ácido indolbutírico $\left(0,1.500,3.000\right.$ ó $\left.4.500 \mathrm{mg} \mathrm{L}^{-1}\right)$, y el segundo factor fue el tamaño de cladodio $(40$ ó $60 \mathrm{~cm})$. El experimento tuvo ocho tratamientos con tres repeticiones, para un total de 24 unidades experimentales (UE). Cada UE estuvo compuesta por diez cladodios de pitaya, por tanto, se utilizaron 240 cladodios.

Se emplearon cladodios maduros de pitaya amarilla de la especie Selenicereus megalanthus Haw (no existen reportes de la variedad o clon cultivado) de un cultivo comercial de 9 años de plantado en el municipio de Miraflores (Boyacá). El sustrato estuvo compuesto de una mezcla de suelo y cascarilla de arroz sin quemar en relación 1:1 $\mathrm{V} / \mathrm{V}$, el cual se depositó en materas con capacidad para $5 \mathrm{~L}$. Los cladodios se recolectaron de la parte basal de las ramas antes de iniciar la floración; luego se sometieron a una inmersión rápida, $20 \mathrm{~s}$, en las soluciones de AIB; solo se sumergieron $2 \mathrm{~cm}$ de la parte basal de cada cladodio; posteriormente, los cladodios se sembraron en el sustrato preparado, enterrando solo $10 \mathrm{~cm}$ de ellos. 
Los riegos se realizaron diariamente y a los $90 \mathrm{~d}$ se midieron las siguientes variables: Porcentaje de enraizamiento: $\mathrm{PE}=$ (cladodios enraizados / cladodios sembrados) x 100; número de raíces; longitud de raíces $=$ sumatoria de la longitud de la totalidad de las raíces; número de brotes; Iongitud de brotes por medición directa con flexómetro; masa fresca (g) y masa seca ( $\mathrm{g}$ ) de raíces y de brotes mediante medición directa en balanza eléctrica de precisión 0,01 g después de someter las plantas a $75^{\circ} \mathrm{C}$ en estufa durante $48 \mathrm{~h}$.

Para el examen de los datos se realizó un anál isis de varianza (Anava) factorial, que determinó diferencias estadísticas; para los factores y tratamientos se utilizó la prueba de comparación de promedios de Tukey, con una confiabilidad del 95\%, que permitió establecer los mejores promedios, todo con el programa SAS v. 8.1e (Cary, N.C).

\section{RESULTADOS Y DISCUSIÓN}

\section{Enraizamiento}

En el porcentaje de enraizamiento no se presentaron diferencias estadísticas entre tratamientos, tamaño del cladodio ni concentración de AIB (tablas 1 y 2); sin embargo, se reporta que el enraizamiento generalmente es distinto con el tipo de estaca utilizada (cladodio en este caso), pues este potencial varía entre las diferentes porciones de un mismo tallo (Hartmann et al., 2002).

Los porcentajes de enraizamiento obtenidos son bajos si se comparan con los encontrados por De Andrade et al. (2007) para pitaya roja, quienes obtuvieron $100 \%$ de prendimiento sin la utilización de ningún tipo de promotor de enraizamiento, concluyendo que se presenta facilidad de enraizamiento de esta especie. Estos resultados pondrían en evidencia la existencia de diferencias en el comportamiento de la propagación asexual entre plantas de una misma familia.

Según Hartmann et al. (2002), la capacidad para regenerar la estructura entera de la planta depende de dos características: la primera es la totipotencia celular, y la segunda es la desdiferenciación celular. Aunque la iniciación de raíces no requiere de la producción de fotoasimilados, sí necesita de las reservas almacenadas y de una acumulación de hormonas de enraizamiento, transportadas basipétalamente (Ryugo, 1993).

Contrario a lo encontrado en el presente estudio, Bastos et al. (2006) determinaron que el tamaño del cladodio afectó significativamente el prendimiento de plantas de pitaya roja, de tal manera que el mayor tamaño $(25 \mathrm{~cm})$ favoreció el enraizamiento; de igual forma, estos autores concluyeron que la aplicación de $3.000 \mathrm{mg} \mathrm{L}^{-1} \mathrm{de}$ AIB generó mayor porcentaje de enraizamiento, comparado con $0 \mathrm{mg} \mathrm{L}^{-1}$ de AIB. Por su parte, ElObeidy (2006) encontró que con estacas de pitaya roja de $25 \mathrm{~cm}$ se produjo enraizamiento a las 2 semanas; no obstante, este autor sugiere que con estacas de $5 \mathrm{~cm}$ y $10 \mathrm{mM}$ de AIB se favorece la propagación de esta especie. Sin embargo, en pitaya amarilla una mayor longitud del cladodio permite que la planta alcance más rápidamente la altura del tutorado y que la distribución de las ramas sobre este sea más rápida y más adecuada; plantas con estas características inician mucho antes la etapa productiva, razón por la cual se espera que con el cladodio de $60 \mathrm{~cm}$ se puedan obtener mayores beneficios.

\section{Número de raíces}

No fue estadísticamente diferente entre tratamientos ni en cada uno de los factores por separado (tablas 1 y 2 ). Se esperaba que los cladodios de $60 \mathrm{~cm}$ presentaran una respuesta más favorable en el número de raíces, debido a que, como lo afirman Bastos et al. (2006), los cladodios de mayor tamaño son más lignificados y han acumulado mayor cantidad de sustancias de reserva (carbohidratos) y hormonas necesarias para inducir mayor número de raíces, tal como fue reportado en Ficus carica L. por Norberto et al. (2001).

La respuesta en enraizamiento es aún mayor cuando se acompaña de la aplicación exógena de AIB, posiblemente, porque el contenido endógeno de auxinas en los tejidos de los cladodios de pitaya puede ser bajo; de esta forma, el suministro de AIB exógeno promueve una alteración hormonal, aumentando los niveles de auxinas en los cladodios, lo cual favorece la producción de raíces 


\begin{tabular}{|l|c|c|c|c|c|c|c|c|c|}
\hline \multicolumn{7}{|c|}{ Tabla 1. Efecto del tratamiento con AIB y tamaño del cladodio sobre la propagación asexual de pitaya. } \\
\hline
\end{tabular}

Promedios con letras distintas indican diferencia significativa según la prueba de Tukey $(P \leq 0,05)$. ns: no hay diferencias; ${ }^{\star \star}$ diferencias estadísticas al $1 \%$; * diferencias estadísticas al 5\%, de acuerdo con el Anava.

(Bastos et al. 2006); sin embargo, esto no se puedo evidenciar de forma clara en esta investigación, debido posiblemente a que altas concentraciones de auxinas pudieron causar inhibición del enraizamiento y crecimiento de raíces (Taiz y Zeiger, 2006).

Fachinello et al. (1995) afirman que la capacidad de una estaca para emitir raíces está en función de factores endógenos y exógenos, por lo cual, la formación de raíces adventicias se debe a la interacción de tales factores, principalmente, una translocación de sustancias localizadas en las hojas o yemas, en donde es el centro de producción de hormonas, que son translocadas vía floema a la estaca. Estas sustancias controlan la división celular, pudiendo ser limitantes en la estimulación de procesos fisiológicos como el enraizamiento de estacas (Torrey, 1996).

Una mayor emisión de raíces por estaca también fue obtenida por Biasi et al. (1997), con mayor concentración de AIB (2.000 $\left.\mathrm{mg} \mathrm{L}^{-1}\right)$, pues en los estadios iniciales de la inducción del enraizamiento, altas concentraciones de auxinas son necesarias para el crecimiento de primordios radicales (Taiz y Zeiger, 2006).

A pesar de que con las estacas de $40 \mathrm{~cm}$ y $\sin \mathrm{AIB}$ no hubo emisión de brotes y que con $4.500 \mathrm{mgL}^{-1}$ de AIB en las estacas de $60 \mathrm{~cm}$ se obtuvieron más de cuatro brotes, esta respuesta no fue significativa (tabla 1). Tampoco se encontraron diferencias estadísticas para el tamaño de cladodio, aunque fue mejor el de $60 \mathrm{~cm}$ (tabla 2), y las dosis de AIB fueron estadísticamente similares.

Mizrahi et al. (2002) sugieren que el tamaño del cladodio más indicado para la propagación de pitaya está entre 25 y $40 \mathrm{~cm}$, sin embargo, en este trabajo se demostró que con cladodios entre 40 y $60 \mathrm{~cm}$ la respuesta es favorable. Al respecto, LópezGómez et al. (2000) evaluaron el efecto de dos tamaños de cladodio $(0,5$ y 1,0 m) en la propagación asexual de pitaya y encontraron que los más largos produjeron mayor cantidad de brotes. 
Tabla 2. Efecto del tamaño de estaca sobre la propagación asexual de pitaya.

\begin{tabular}{|c|c|c|c|c|c|c|c|c|c|}
\hline \multirow{2}{*}{$\begin{array}{l}\text { Tamaño de } \\
\text { estaca }(\mathrm{cm})\end{array}$} & \multirow{2}{*}{$\begin{array}{c}\text { Enraizamiento } \\
(\%)\end{array}$} & \multicolumn{2}{|c|}{ Longitud (cm) } & \multicolumn{2}{|c|}{ Número } & \multicolumn{2}{|c|}{ Masa fresca (g) } & \multicolumn{2}{|c|}{ Masa seca (g) } \\
\hline & & Raíces & Brotes & Raíces & Brotes & Raíces & Brotes & Raíces & Brotes \\
\hline 40 & $49,17 a$ & $2,14 b$ & $3,11 \mathrm{a}$ & $5,63 a$ & $1,98 a$ & $2,39 b$ & $0,84 b$ & $0,26 a$ & $0,08 a$ \\
\hline 60 & $50,83 a$ & $6,79 a$ & $4,36 a$ & $6,72 a$ & $2,72 a$ & $3,63 a$ & $2,42 a$ & $0,43 a$ & $0,16 a$ \\
\hline Significancia & ns & ** & ns & ns & ns & * & ** & ns & ns \\
\hline
\end{tabular}

Promedios con letras distintas indican diferencia significativa según la prueba de Tukey $(P \leq 0,05)$. ns: no hay diferencias; ${ }^{* *}$ diferencias estadísticas al $1 \%$; * diferencias estadísticas al 5\%, de acuerdo con el Anava.

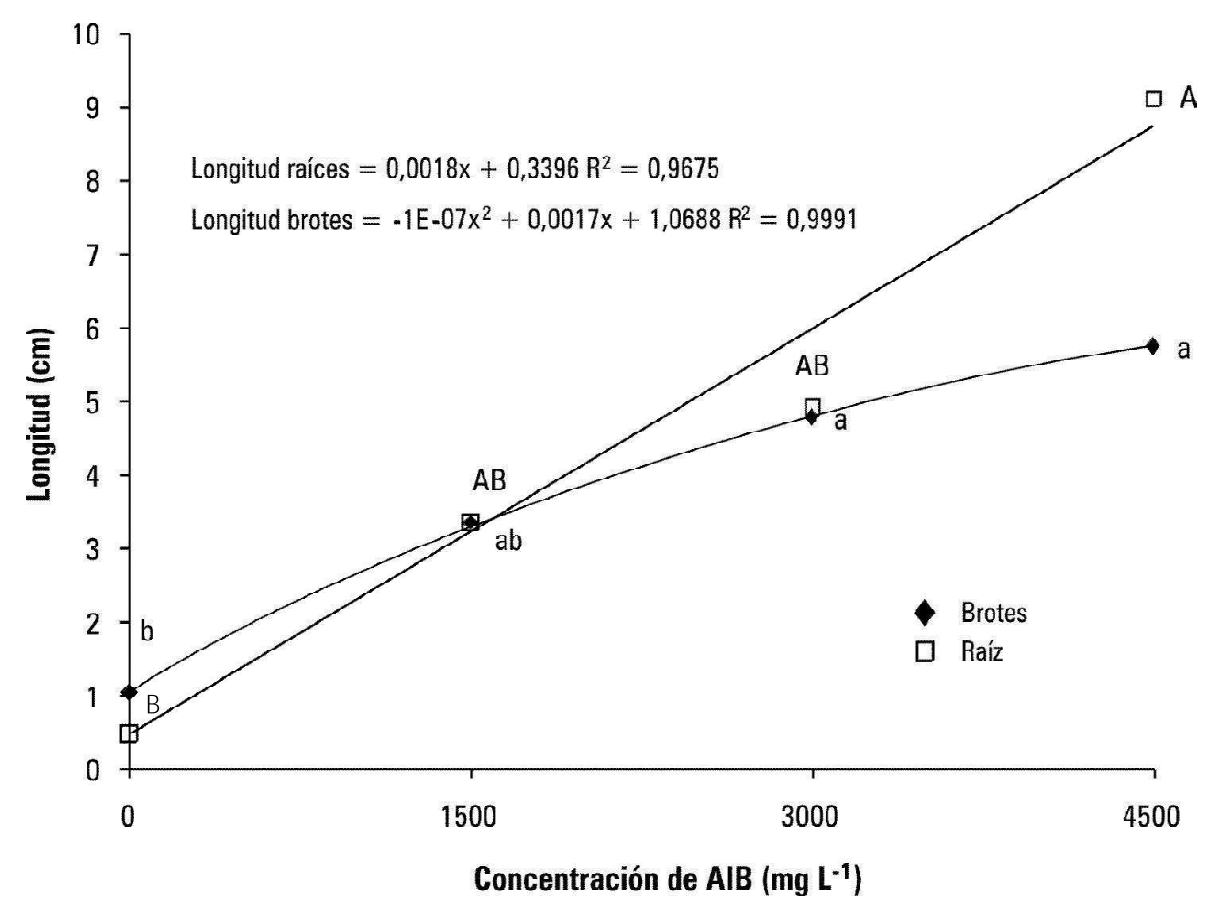

Figura 1. Efecto de la concentración de AIB sobre la longitud de raíces de pitaya propagada asexualmente. Promedios con letras distintas indican diferencia significativa según la prueba de Tukey $(P \leq 0,05)$.

\section{Longitud de raíces y brotes}

La longitud de raíces fue estadísticamente diferente debido a los tratamientos, al efecto independiente del AIB y al tamaño del cladodio. El mejor tratamiento fue el cladodio de $60 \mathrm{~cm}$ y la concentración de $4.500 \mathrm{mg} \mathrm{L}^{-1}$ de AIB (tabla 1); estos factores, de forma independiente, también generaron la mayor respuesta (tabla 2, figura 1); también se observó un aumento lineal de la longitud de raíces conforme se incrementó la concentración de AIB (figura 1).

Según Hartmann et al. (2002), las auxinas son las sustancias más importantes en el proceso de enraizamiento de estacas, dado que entre sus principales funciones biológicas se destaca el crecimiento de órganos, principalmente de raíces, razón por la cual, con la aplicación exógena de 
AIB se esperaría que se favoreciera el crecimiento en longitud de las raíces de pitaya. Santelices (2007) encontró mayor enraizamiento en estacas de Nothofagus glauca (Phil.) a medida que aumentó la concentración de AIB de 0,5\% a 1,5\%; sin embargo, no se apreció un efecto claro de la auxina en la longitud de las raíces, este último resultado difiere con lo encontrado para pitaya en el presente experimento.

Se obtuvo mayor longitud de brotes con el tratamiento de $4.500 \mathrm{mg} \mathrm{L}^{-1}$ de AIB en los cladodios de $60 \mathrm{~cm}$, la menor respuesta fue sin AIB en las estacas de $40 \mathrm{~cm}$ (tabla 1). El análisis estadístico de factores indicó que hubo diferencias estadísticas entre las dosis de AIB y que la mejor fue $4.500 \mathrm{mg} \mathrm{L}^{-1}$ (figura 1), mientras que entre tamaños de cladodio no hubo diferencias significativas (tabla 2).

El crecimiento del tallo se da por acción directa de las auxinas o por el aumento que generan en la biosíntesis de giberelinas (Taiz y Zeiger, 2006), pues estas dos hormonas tienen como efecto fisiológico la elongación celular, que se traduce en mayor longitud del tallo (en este caso los brotes). Además, las giberelinas pueden aumentar la longitud por la interacción de tres eventos: el primero consiste en la estimulación de la división celular en el ápice del tallo; el segundo comprende la promoción del crecimiento celular, ya que incrementan la hidrólisis del almidón, fructanos y sacarosa, originando moléculas de glucosa y fructosa, y en el tercero se aumenta la plasticidad de la pared celular (Salisbury y Ross, 1994).

\section{Masa fresca}

Hubo mayor producción de masa fresca de brotes, y con diferencias estadísticas, con el tratamiento correspondiente a los cladodios de $60 \mathrm{~cm}$ y la inmersión en $4.500 \mathrm{mg} \cdot \mathrm{L}^{-1}$ de AIB (tabla 1). Respecto a las dosis de AIB, se presentaron diferencias estadísticas, además, el aumento de masa fresca siguió una tendencia de una función cuadrática (figura 2). Los cladodios de $60 \mathrm{~cm}$ fueron superiores a los de $40 \mathrm{~cm}$ en la masa fresca de brotes $(P \leq 0,05)$ (tabla 2$)$.

Se observó mayor masa fresca de raíces en las estacas de $60 \mathrm{~cm}$ con la adición de $4.500 \mathrm{mg} \mathrm{L}^{-1}$ de AIB $(P \leq 0,05)$ (tabla 1); además, esta masa fresca se vio favorecida con los incrementos del

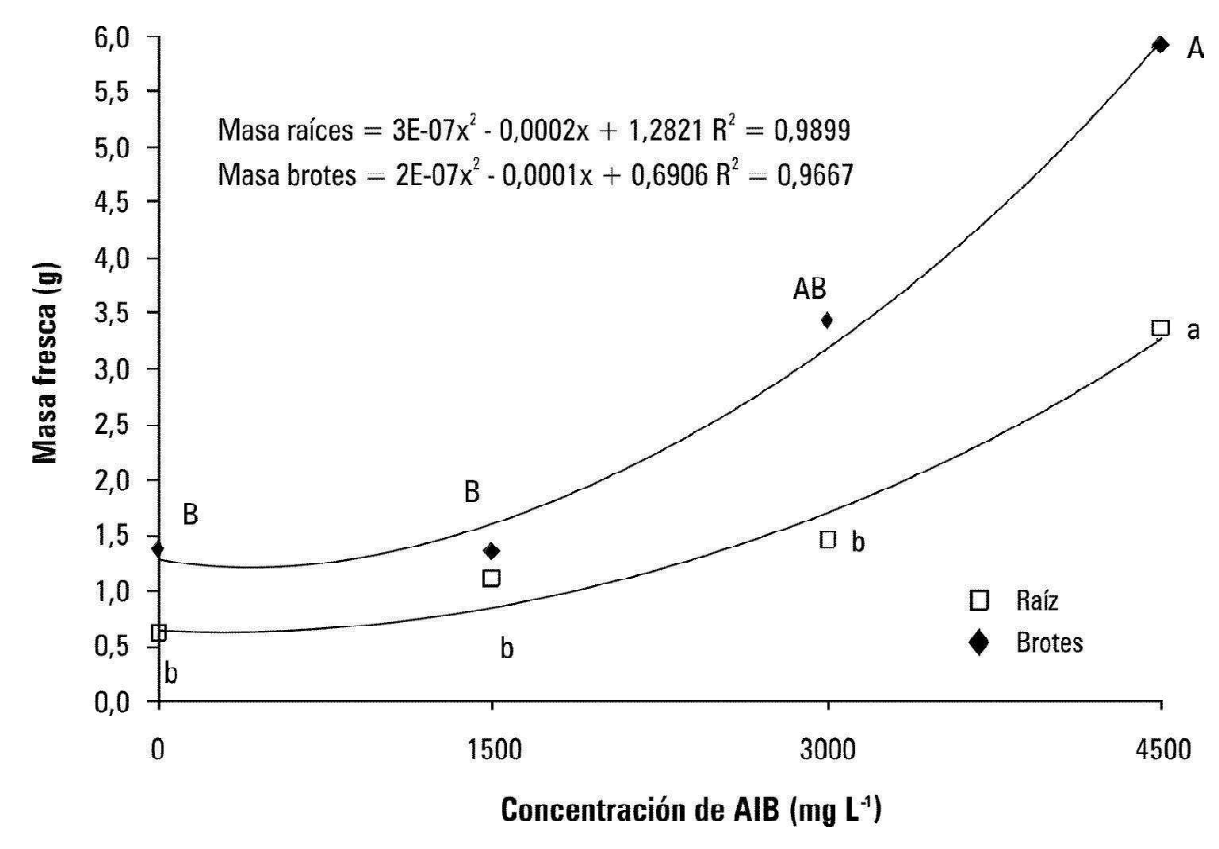

Figura 2. Efecto de la concentración de AIB sobre la masa fresca de brotes y raíces de pitaya propagada asexualmente. Promedios con letras distintas indican diferencia significativa según la prueba de Tukey $(P \leq 0,05)$. 
AlB $(P \leq 0,05)$ y se ajustó a un polinomio de segundo grado (figura 2). Los cladodios de $60 \mathrm{~cm}$ generaron mayor masa fresca de raíces $(P \leq 0,05)$ (tabla 2).

Una mayor acumulación de masa fresca en la raíces depende, entre otros factores, de un sistema radical de gran tamaño (mayor número y longitud de raíces), capaz de acumular reservas y agua en mayor proporción. Es posible que con mayor contenido de auxinas en los cladodios de pitaya se aumente la elongación celular, que se da porque las auxinas provocan que las células receptoras secreten $\mathrm{H}^{+}$en las paredes primarias circundantes, los cuales reducen el $\mathrm{pH}$, de tal forma que la pared pierde rigidez por acción de expansinas, proteínas que provocan la distensión de las paredes celulares por debilitamiento de los puentes de hidrógeno entre los polisacáridos y los componentes de la pared celular; posteriormente, la célula se expande por incorporación osmótica de agua (Taiz y Zeiger, 2006), Io cual se traduce en mayor contenido hídrico de la planta y también en mayor masa fresca.

La mayor masa fresca de brotes, obtenida por acción del tratamiento de $60 \mathrm{~cm}$ y la inmersión en $4.500 \mathrm{mg} \mathrm{L}^{-1}$ de AIB, se debió, posiblemente, a que se generó un mayor sistema radicular capaz de captar más nutrientes y agua, y de esta manera generar mayor masa; así mismo, la mayor cantidad de reservas presentes en el cladodio de $60 \mathrm{~cm}$ también pudo estimular mayor cantidad de brotes y de mayor tamaño. Estos brotes, posteriormente, se van a convertir en órganos fuente capaces de realizar fotosíntesis y producir fotoasimilados para el desarrollo de la planta, los cuales, muy seguramente, favorecerán en mayor grado la producción de más frutos y de un tamaño más grande.

\section{Masa seca}

La masa seca de raíces no se afectó significativamente por los tratamientos (tabla 1). Respecto a los factores de forma independiente, se observó un efecto significativo del AIB, el cual generó una respuesta lineal y directamente proporcional a medida que se aplicó en mayor concentración (figura 3); el tamaño del cladodio no fue significativo para esta variable (tabla 2).
Lo resultados obtenidos con la aplicación de AIB para pitaya en este estudio confirman Io encontrado por Zietemann y Roberto (2007), quienes demostraron que en la propagación de guayaba cultivares 'Paluma' e 'Século $X X I$ ' mediante estacas se presentó mayor masa seca de raíces con la aplicación de $800 \mathrm{mg} \mathrm{L}^{-1}$ de AIB respecto a dosis menores. Igual tendencia se encontró en el enraizamiento de estacas de Malpighia glabra L., aunque la mayor masa seca de raíces se logró con $2.800 \mathrm{mg} \mathrm{L}^{-1}$ de AIB, mientras que en acodos de Murraya paniculata se obtuvo mayor masa seca, fresca y longitud total de raíces con solo $4 \mathrm{mg} \mathrm{L}^{-1}$ de AIB (Castillo et al., 2005); esto pone de manifiesto la importancia biológica de la aplicación exógena de AIB en la propagación asexual de diferentes especies.

Hubo mayor repuesta en la masa seca de brotes con la aplicación de $4.500 \mathrm{mg} \mathrm{L}^{-1}$ de AIB en los cladodios de $60 \mathrm{~cm}(P \leq 0,05)$ (tabla 1). Se presentó un aumento cuadrático y significativo de la masa seca de brotes en función de la concentración de AIB (figura 3), pero no se observaron diferencias estadísticas entre tamaños de cladodio; no obstante, el cladodio de $60 \mathrm{~cm}$ favoreció en mayor grado la masa seca de los brotes (tabla 2).

Con los resultados obtenidos queda demostrado que las auxinas ejercen una importante función sobre el crecimiento y desarrollo vegetal, incluyendo la emisión de brotes aéreos y la estimulación del enraizamiento de cladodios, cuando son aplicadas exógenamente, lo cual es constatado por Hartmann et al. (2002).

Es evidente que un cladodio de mayor tamaño y un sistema radical más vigoroso producido por la aplicación exógena de AIB van a originar mayor síntesis de carbohidratos o la removilización de estos desde el cladodio a los nuevos brotes, con lo cual la masa seca va a ser mayor. Una disponibilidad de carbohidratos es considerada factor limitante de sobrevivencia de estacas, pues presenta la principal fuente de energía asimilable para el enraizamiento y mantenimiento de las actividades metabólicas de las estacas (Veierskov, 1988); por lo cual, los cladodios de pitaya de 60 $\mathrm{cm}$ tratados con la mayor concentración de AIB van a continuar con un crecimiento vigoroso por tener alta disponibilidad de energía y carbono estructural para la formación de nuevos tejidos. 


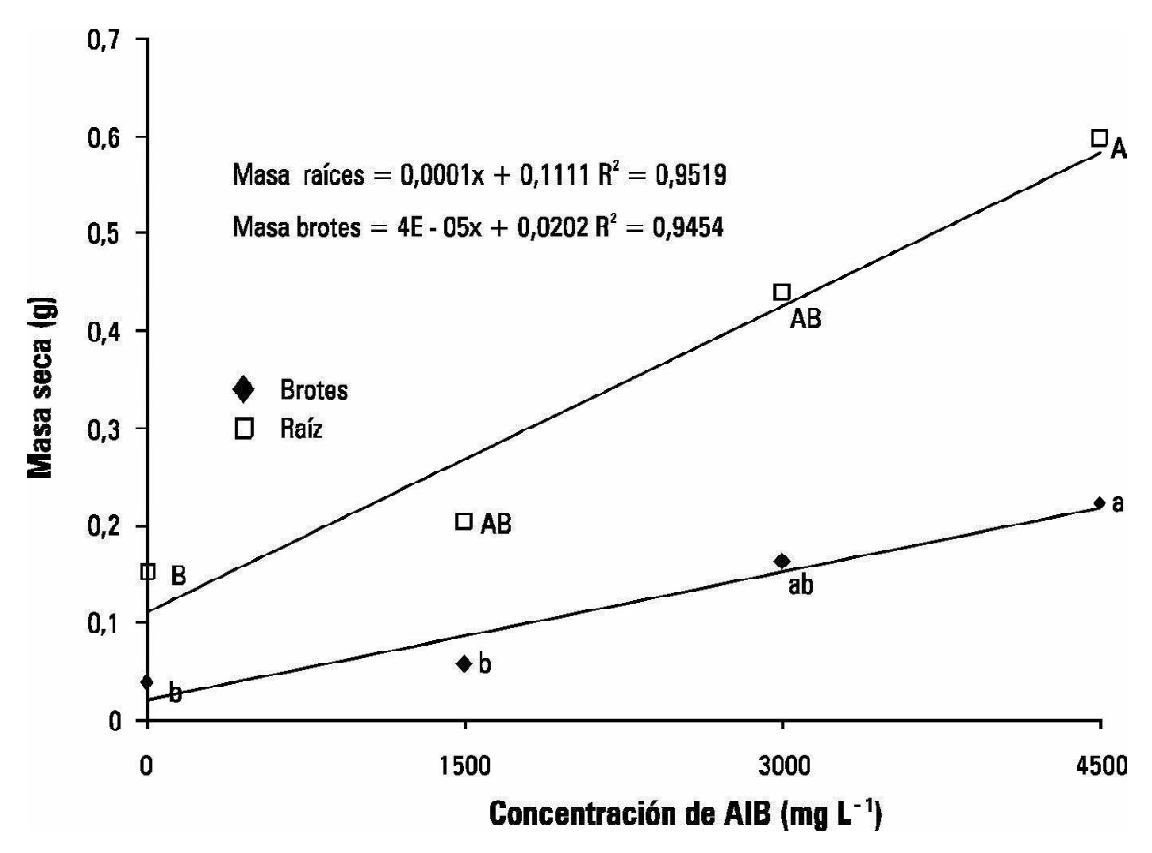

Figura 3. Efecto de la concentración de AIB sobre la masa seca de raíces y brotes de pitaya propagada asexualmente. Promedios con letras distintas indican diferencia significativa según la prueba de Tukey $(P \leq 0,05)$.

\section{CONCLUSIONES}

Se concluye que la propagación asexual de pitaya es fisiológicamente más conveniente mediante la inmersión de cladodios de $60 \mathrm{~cm}$ en $4.500 \mathrm{mg}$ $\mathrm{L}^{-1}$ de $\mathrm{AIB}$; esto garantiza mayor prendimiento, un sistema radical de mejor calidad y mayor producción de brotes.

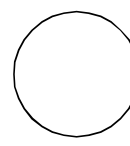

Agronet. 2010. Producción y área cosechada de Pitaya en Colombia en 2008. Disponible en: http:// www.agronet.gov.co/; consulta: junio de 2010.

Bastos, D.; R. Pio; J.A. Scarpare; M. Neubern; L.F. Paes De Almeida; T.P. Dias y S. Bakker. 2006. Propagation of red pitaya (Hylocereus undatus) by cuttings. Ciênc. Agrotec. 30(6), 1106-1109.

Biasi, L. A.; C.V. Pommer y P.A. Pino. 1997. Propagação de porta-enxertos de videira mediante estaquia semilenhosa. Bragantia 56(2), 367-376.

Castillo M.; Y.H. De Fréitez y N. Hernández. 2005. efecto de la auxina AIB en la propagación de azahar de la india (Murraya paniculata (L. Jack)) por acodo aéreo. Bioagro 17(2), 123-126.

\section{REFERENCIAS BIBLIOGRÁFICAS}

CCl. Corporación Colombia Internacional. 2008. La pitaya. En: www.cci.org.co; consulta: enero de 2009

Celenza, J.L.; P. L. Grisafi y G.R. Fink. 1995. A pathway for lateral root formation in Arabidopsis thaliana. Genes Dev. 9, 2131-2142.

Costa, J M. y H. Challa. 2002. The effect of the original leaf area on growth of softwood cuttings and planting material of rose. Scientia Hort. 95, 111121.

De Andrade R.A.; A.B. Geraldo y M.T. Habib. 2007. Influência da fonte de material e do tempo de cura na propagação vegetativa da pitaya vermelha (Hylocereus undatus Haw). Rev. Bras. Frutic. 29(1), 183-186. 
Dueñas, Y.; C.E. Narváez y L.P. Restrepo. 2008. Inhibición de lesiones por frío de pitaya amarilla (Acanthocereus pitajaya) a través del choque térmico: catalasa, peroxidasa y polifenoloxidasa. Acta Biol. Colomb. 13(1), 95 -106.

ElObeidy, A.A. 2006. Mass propagation of pitaya (dragon fruit). Fruits 61(5), 313-319.

Ermel, F.F.; S. Vizoso; J. P. Charpentier; C. Jay-Allemand; A.M. Catesson e I. Couée. 2000. Mechanisms of primordium formation during adventitious root development from walnut cotyledon explants. Planta 211, 563-574.

Fachinello, J.C.; A. Hoffmann; J.C. Nachtigal, E. Kersten y G.R. Fortes. 1995. Propagação de plantas frutíferas de clima temperado. 2a. ed. Universidad Federal de Pelotas. Pelotas, Brasil.

Hartmann, H. T.; D. E. Kester; F.T. Davies Junior y R.L. Geneve. 2002. Plant propagation: principles and practices. 7. ed. Prentice Hall, Upper Saddle River, NJ.

Hinojosa, G.F. 2000. Auxinas. pp. 15-54. En: Cid, L.P.B. (ed.) Introdução aos hormônios vegetais. Embrapa, Brasília, DF.

Li, S.W.; L.G. Xue; S. J. Xu; H.Y. Feng y L Z. An. 2009. IBA-induced changes in antioxidant enzymes during adventitious rooting in mung bean seedlings: The role of $\mathrm{H}_{2} \mathrm{O}_{2}$. Environ. Exp. Bot. 66, 442-450

López-Gómez, R.; J.C. Díaz-Pérez y G. Flores-Martínez. 2000. Propagación vegetativa de tres especies de cactáceas: Pitaya (Stenocereus griseus), Tunillo (Stenocereus stellatus) y Jotilla (Escontria chiotilla). Agrociencia 34(4), 363-367.

Mizrahi, Y.; A. Nerd e Y. Sitrit. 2002. New fruits for arid climates. pp. 378-384. En: Janick, J. y A. Whipkey (eds.). Trends in new crops and new uses. Alexandria, Egipto.

Moreno, N.; J.G. Álvarez-Herrera; H.E. Balaguera-López y G. Fischer. 2009. Propagación asexual de uchuva
(Physalis peruviana L.) en diferentes sustratos y a distintos niveles de auxina. Agron. Colomb. 27(3), 341-348.

Norberto, P.M.; N. N. J. Chalfun; M. Pasqual; R.D. Veiga; G.E. Pereira y J.H. Mota. 2001. Efeito da época de estaquia e do AIB no enraizamento de estacas de figueira (Ficus carica L.). Ciênc. Agrotec. 25(3), 533-541.

Pasqual, M.; N.N. J. Chalfun; J.D. Ramos; M.R. do Vale y C.R. de Silva. 2001. Fruticultura comercial: propagação de plantas frutíferas. Universidad Federal de Lavras, Brasil.

Ryugo, K. 1993. Fruticultura - Ciencia y arte. AGT Editor, México, DF.

Salisbury, F. y C. Ross. 1994. Fisiología vegetal. Grupo Editorial Iberoamérica S.A., México, DF.

Santelices, R. 2007. Efecto del ácido indolbutírico (AIB) $\mathrm{y}$ de la presencia de hojas en el arraigamiento de estacas de Nothofagus glauca (Phil.) Krasser cosechadas en dos épocas diferentes. Ecología Austral 17, 151-158.

Taiz L. y E. Zeiger. 2006. Plant physiology. $4^{\text {th }}$ ed. Sinauer Associates, Inc., Publishers, Sunderland, MA

Torrey, J.G. 1996. Endogenous and exogenous influences on the regulation of lateral root formation. pp. 31-66. En: Jackson, M.B. (ed.). New root formation in plants and cuttings. M. Nijhoff, Dordrecht, The Netherlands.

Veierskov, B. 1988. Relations between carbohydrates and adventitious root formation. pp.70-78. En: Davies, T.D.; B.E. Haissig y N. Sankla (eds.) Adventitious root formation in cuttings. Dioscorides Press, Portland, OR.

Zietemann, C. y S. R. Roberto. 2007. Efeito de diferentes substratos e épocas de coleta no enraizamento de estacas herbáceas de goiabeira, cvs. Paluma e Século XXI. Rev. Bras. Frutic. 29(1), 31-36. 